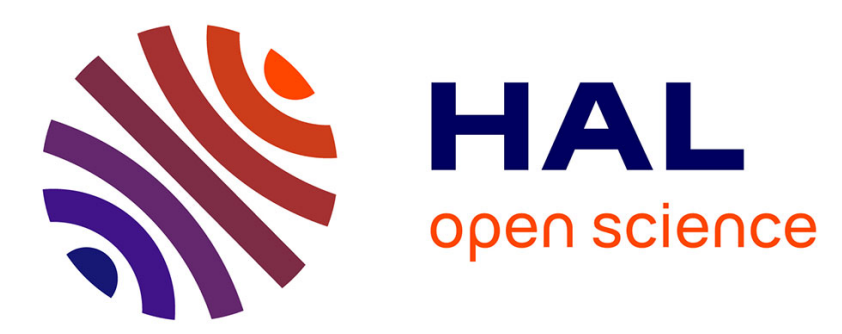

\title{
Influence de l'alignement des fibres sur la rupture des composites en compression sens fibre
}

Gabriel Eyer, Olivier Montagnier, Jean-Paul Charles, Frédéric Mazerolle, Christian Hochard

\section{- To cite this version:}

Gabriel Eyer, Olivier Montagnier, Jean-Paul Charles, Frédéric Mazerolle, Christian Hochard. Influence de l'alignement des fibres sur la rupture des composites en compression sens fibre. Matériaux \& Techniques, 2016, 104 (4), pp.404. 10.1051/mattech/2016023 . hal-02319408

\section{HAL Id: hal-02319408 https://hal.science/hal-02319408}

Submitted on 9 Dec 2019

HAL is a multi-disciplinary open access archive for the deposit and dissemination of scientific research documents, whether they are published or not. The documents may come from teaching and research institutions in France or abroad, or from public or private research centers.
L'archive ouverte pluridisciplinaire HAL, est destinée au dépôt et à la diffusion de documents scientifiques de niveau recherche, publiés ou non, émanant des établissements d'enseignement et de recherche français ou étrangers, des laboratoires publics ou privés. 


\section{Influence de l'alignement des fibres sur la rupture des composites en compression sens fibre}

\author{
Gabriel Eyer $^{1}$, Olivier Montagnier ${ }^{1,2}$, Jean-Paul \\ Charles $^{1}$, Frédéric Mazerolle ${ }^{1}$, Christian Hochard
}

1 : Aix Marseille Univ, CNRS, Centrale Marseille, LMA, UPR
7051, Marseille, France
4 impasse Nikola Tesla, CS 40006, 13453 Marseille Cedex 13,
France
e-mail : eyer@lma.cnrs-mrs.fr, jean-paul.charles@univ-amu.fr, mazerolle@Ima.cnrs-mrs.fr, christian.hochard@univ-amu.fr

2: Centre de recherche de l'Armée de l'air (CReA) École de l'air (EA), BA 701, 13361 Salon Air, France e-mail : olivier.montagnier@defense.gouv.fr

Résumé - Ce travail s'intéresse à la mesure et à la modélisation de la résistance en compression sens fibre pour des composites unidirectionnels carbone/époxy, et plus particulièrement, à l'influence de l'alignement global des fibres. Dans un premier temps, à partir de résultats expérimentaux précédents, il est montré qu'en présence d'endommagement diffus dans la matrice, celui-ci abaisse significativement la résistance en compression sens fibre. Une modélisation à l'échelle méso basé sur une variable d'endommagement de la matrice est donnée pour rendre compte du phénomène. Dans un second temps, ces résultats sont appliqués au cas de la compression avec des éprouvettes présentant un défaut d'alignement. II sera montré que, dans ce cas, le problème devient complexe puisqu'il couple alignement des fibres et endommagement matriciel. A partir d'une expérience originale de compression sur éprouvette avec concentration de contrainte, nous montrons l'intérêt de prendre en compte l'état d'endommagement de la matrice pour la prédiction de la rupture.

Mots-clés : Rupture, Compression, Composite, Endommagement, Alignement des fibres

Abstract - Effect of fibers alignment on the failure of composites in compression in the fiber direction. This work deals with the measurement and modeling of compressive strength in the fiber direction for unidirectional carbon/epoxy composite, and focuses on the influence of the global misalignment angle of the fibers. At first, from previous experimental results, it is shown that the presence of diffuse damage in the matrix significantly modify the compressive strength. A mesoscale model based on matrix damage variable is given to account for the phenomenon. Secondly, these results are applied to the case of specimens with global misalignment angle. It will be shown that in this case, the problem becomes complex since misalignment of the fibers and matrix damage are coupled. Finally, from an original compression test specimens with stress concentration, we show the interest to take into account the matrix damage state for predicting the failure.

Key words: Failure, Compression, Composite, Damage, Fiber misalignment

\section{Introduction}

Le dimensionnement des structures composites passe par des modèles numériques toujours plus complexes dont la qualité découle de la capacité à reproduire le comportement du matériau. Pour les composites stratifiés, le comportement en compression dans la direction des fibres s'avère complexe et reste encore mal connu. Une des principales difficultés pour ce type de sollicitation est le lien entre la partie expérimentale, la mise en place des modèles et leur validation.

Hochard et al. [1] ont déjà montré expérimentalement pour des sollicitations de traction que l'augmentation de l'endommagement transverse avait pour conséquence de diminuer la résistance du pli. Il est pressenti que l'endommagement joue un rôle d'autant plus important pour la compression. Lorsque la résine est intégralement endommagée, l'élancement des fibres mène à leur flambage immédiat, ce qui implique une rupture catastrophique du pli. Gibson et al. [2] ont montré que l'augmentation de la température (ce qui s'apparente à un endommagement de la matrice) entraînait une chute de la résistance en compression pour des composites verre/polypropylène. Et lorsque la température atteint le seuil de transition vitreuse, le pli présente une résistance quasi-nulle.

De nombreux auteurs [3, 4, 5, 6] considèrent que la rupture en compression correspond à une instabilité à l'échelle des fibres connue sous le nom de microflambage. Le microflambage élastique intervient en premier lieu au voisinage d'une imperfection (amas de résine, fibre rompue, désalignement local, etc...). Ensuite l'état post-flambé des fibres engendre des déformations trop importantes pour les fibres qui finissent par se rompre. Cela génère ensuite des bandes de glissement (kink-band, figure 1 [7]).

Nombre de modèles micros visent à décrire la rupture du pli en compression, notamment via la modélisation de ces bandes de glissement $[4,5,6]$. Ces modélisations se complètent avec la mécanique de l'endommagement dans [8].

Rosen [3] prévoit que le flambage élastique des fibres est directement lié au module de cisaillement de la matrice $G_{m}$ : 


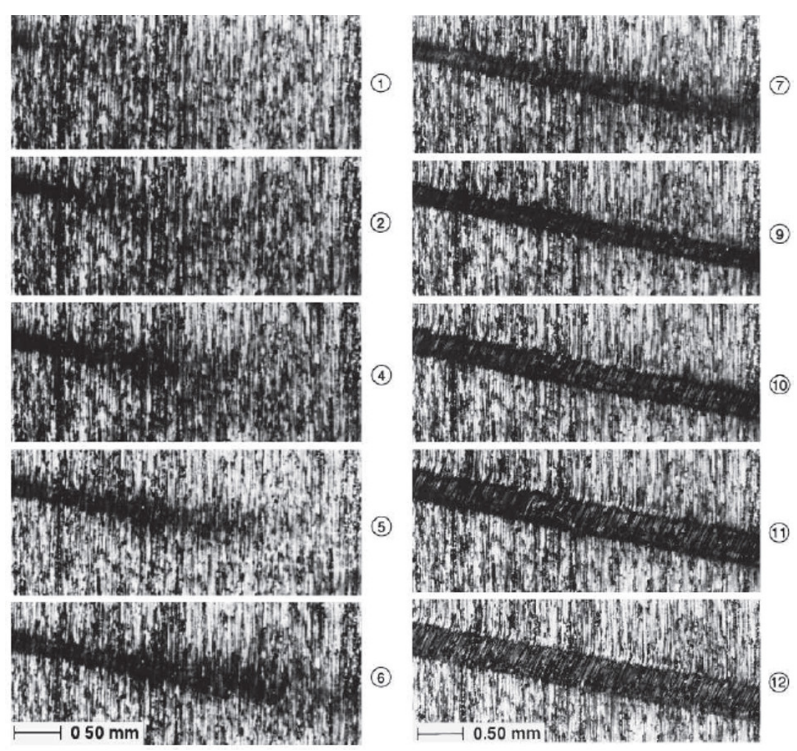

Figure 1 - Apparition d'une kink-band [7]

Fig. 1. Appearance of a kink-band [7]

$$
\sigma_{\min }=\frac{G_{m}}{1-V_{f}}
$$

où $V_{f}$ est le taux de fibres. Cette relation surestime très largement la contrainte à rupture et est insensible à un défaut de désalignement local des fibres. Budiansky [4] propose plutôt de modéliser une bande de glissement. II constate qu'en considérant un comportement elasto-plastique parfait pour la matrice, la situation change fondamentalement et obtient le critère d'instabilité suivant :

$$
\sigma_{m i n}=\frac{G_{12}}{1+\frac{\bar{\phi}}{\gamma_{Y}}}
$$

où $\bar{\phi}$ représente le désalignement local des fibres, et $\gamma_{Y}$ le glissement élastique maximal de la matrice. D'autres micro-modèles existent dans la littérature, la figure 2 montre l'évolution de quelques-uns en fonction du défaut d'alignement $[9,5,10,11]$. Hormis le modèle de Lo et Chim [9], ces micro-modèles montrent un impact très fort de l'alignement des fibres. Une fois homogénéisé avec des répartitions aléatoires de défauts, l'effet du défaut d'alignement est beaucoup plus faible en particulier autour de $0^{\circ}$ [11]. Il est effectivement montré numériquement que si l'on modélise un ensemble de fibres dont seulement quelques unes sont désalignées, la contrainte à rupture n'est pas directement liée au microflambage de la fibre la plus inclinée [12]. Cela se justifie pas le fait que les fibres adjacentes à la fibre la plus inclinée, qui ne possède pas le même défaut d'alignement, vont jouer un rôle stabilisateur [13].

En définissant classiquement [14] le lien entre le

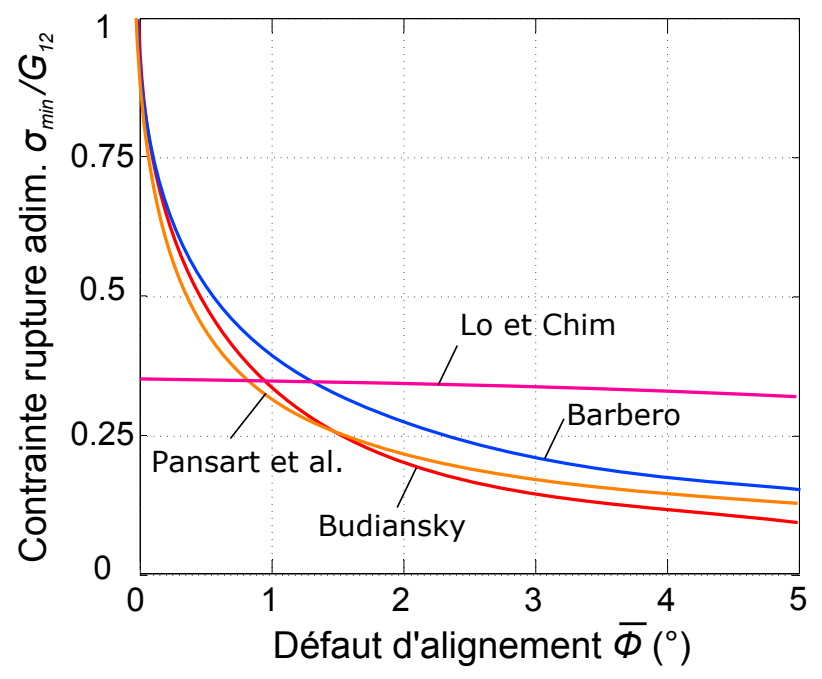

FiguRE 2 - Évolution de la contrainte de rupture en compression adimensionnée en fonction du désalignement local des fibres pour différents modèles de microflambage $[9,4,10,11]$

Fig. 2. Evolution of dimensionless compressive stress versus local fiber misalignment for various microbuckling models $[9,4,10,11]$

module de cisaillement et la variable d'endommagement $d$ par la relation :

$$
G_{12}=G_{12}^{0}(1-d)
$$

les micro-modèles de Rosen et de Budiansky (Eqs. (1-2)) montrent que l'endommagement va jouer un rôle important dans la rupture en compression. Le modèle de Budiansky (Eq. (2)) montre de plus que la contrainte à rupture est significativement affectée par le désalignement local des fibres. Si maintenant on s'intéresse à la compression d'une éprouvette dont les fibres sont globalement désalignées par rapport à l'axe de compression, il va résulter un cisaillement de la matrice qui aura pour effet de créer de l'endommagement et qui entrainera là aussi une rupture prématurée.

II semble donc que ces phénomènes soient liés. C'est dans ce cadre que nous proposons une étude expérimentale qui tente de mettre en évidence le rôle de l'endommagement de la matrice dans la rupture en compression sens fibre pour des éprouvettes présentant un défaut d'alignement global des fibres. Par rapport à la littérature qui précède, l'approche proposée se situera à l'échelle méso. Dans un premier temps, le choix des essais est expliqué a travers une revue de littérature. Dans une seconde partie, l'analyse expérimentale se portera sur l'effet de l'endommagement sur le comportement en compression sens fibre. Enfin, une seconde étude expérimentale sera proposée afin de déterminer la baisse de résistance due au mauvais alignement global des fibres. 


\section{Choix des essais}

Même si ce papier se focalise sur l'influence d'un défaut d'alignement global des fibres sur la rupture en compression, le cadre général de ce travail concerne l'influence de l'endommagement transverse sur le comportement en compression sens fibres $[15,16]$. Pour répondre à cette problématique, il a été nécessaire de proposer des méthodes et des essais permettant à la fois d'endommager la matrice et de réaliser la compression sens fibre. Deux voies sont possibles. La première consiste à proposer un essai permettant de réaliser à la fois l'endommagement de la matrice et la compression sens fibre. La seconde consiste à utiliser deux moyens d'essai séparés.

Parmi les essais permettant de réaliser de la compression, le montage classique, proposé par les normes ASTM D 3410/A et EN ISO 14126, est l'essai Celanese. II est utilisé dans de nombreuses publications $[17,18,19]$. L'avantage principal de cet essai est la géométrie particulièrement simple des éprouvettes et l'accès trivial au champ de contraintes dans l'éprouvette. Cependant les résultats présentent une variabilité importante notamment du fait d'une structure qui est très propice au flambage et qui possède une concentration de contraintes importante au niveau de l'encastrement $[20,17]$. Dans le but de palier à ces défauts, certains auteurs ont proposés de modifier la géométrie de l'éprouvette $[19,21]$ ou d'équiper le montage d'un dispositif externe, dit " anti-flambage ॥ $[2,22]$. Malgré ces améliorations, la déformation à rupture reste faible et présente une variabilité importante. Par conséquent, ces essais nous ont semblé peu propice a une étude fine de l'effet de l'endommagement.

Une autre piste pour caractériser le comportement en compression est d'étudier la face comprimée des essais de flexion, connus pour offrir une meilleure stabilité. Dans cet esprit, on retrouve les essais de flexion 3 points [23], les essais de flexion 4 points $[24,25,26,27]$, les essais de flexion pure [17] et les essais de flambage rotulé [28].

Les essais de flexion avec points d'appuis donnent généralement des valeurs de déformation à rupture élevées. Cependant ils présentent deux difficultés :

- le calcul de l'état de contrainte à partir de l'effort nécessite un calcul inverse complexe lié au grand déplacement de l'éprouvette,

- les points d'appuis peuvent conduire à une rupture prématurée de l'éprouvette.

Montagnier et al. [17] puis Bois et al. [29] ont proposé un essai de flexion pure avec éprouvettes en forme d'haltère dans le but de contourner ces difficultés. Le suivi de l'essai à moment imposé per- met un passage simple du moment de flexion à la contrainte de compression. De plus, la forme haltère à grand rayon permet de localiser la rupture au centre de l'éprouvette. Ce type d'essai permet d'obtenir des déformations à rupture très importantes.

Enfin, Wisnom et al. [28] proposent un essai de flambage rotulé qui permet aussi d'atteindre des déformations à rupture très importantes. Le problème de ce type d'essai est la difficulté à relier l'effort imposé à la contrainte dans la zone utile.

Notons de plus que les essais de flexion mettent en jeu un phénomène de structure complexe lié au gradient de déformation dans l'épaisseur de l'éprouvette $[30,28,23]$.

La stabilité et la précision obtenues par les essais de flexions semblent a priori appropriés à l'étude proposée. En revanche, les éprouvettes de flexion doivent être particulièrement longues et il nous a semblé complexe de pré-endommager (en cisaillement par exemple) ce type d'éprouvette.

Finalement, nous avons proposé deux essais. Le premier consiste a réaliser des éprouvettes tubulaires constituées de plis tissés équilibrés positionnés à $0^{\circ}$ par rapport à l'axe du tube. La forme tubulaire permet de réaliser à la fois l'endommagement en cisaillement par un essai de torsion puis de réaliser la compression. Cela permettra une mesure avec un champ homogène et donc un calcul inverse simple. Ce type d'éprouvette avait déjà été utilisées par Hochard et al. [1] et Miot [31] pour réaliser des essais de cisaillement/traction. Ce premier essai permettra de montrer que l'endommagement affecte sérieusement la résistance en compression sens fibre $[16,15]$. Le second essai consiste à réaliser un essai de compression sur des éprouvettes constituées de plis unidirectionnels positionnés à $0^{\circ}$ et $90^{\circ}$, présentant un élancement faible (faible longueur et épaisseur importante) et une concentration de contraintes. Ce second essai sera utilisé pour déterminer la baisse de résistance due au mauvais alignement des fibres.

\section{Influence de l'endommagement sur la rupture en compression sens fibre}

\subsection{Méthode}

Comme il a été précisé précédemment, les premiers essais sont réalisés sur des tubes en forme d'haltère en tissu carbone/époxy [32]. L'essai se scinde en deux étapes principales (figure 3). Durant la première étape, l'éprouvette tubulaire est soumise à un chargement cyclique de torsion suivi par corrélation 
d'images 3D [33]. II résulte de ce chargement un cisaillement $\sigma_{12}$ des plis tissés et donc un endommagement matriciel. Un pilotage adéquat permet d'atteindre des endommagements importants. La mesure de l'endommagement est obtenue à partir d'un cycle complet de torsion, réalisé à basse vitesse. Sur la figure 4 , la contrainte équivalente de cisaillement (couple de torsion divisé par la surface transversale et le rayon moyen) est tracée en fonction de la déformation de cisaillement (mesurée par corrélation). Le module de cisaillement est mesuré sur ces courbes après linéarisation. L'endommagement $d$ est ensuite aisément déduit de l'équation (3). Plusieurs tubes sont réalisés et les valeurs d'endommagement obtenues sont $0.25,0.5,0.63$ et 0.95 mais seulement trois cycles ont été tracés sur la figure pour une meilleure lisibilité. Suite à cette étape, un travail nécessaire consiste à aligner les fibres dans la direction de la sollicitation de compression. Cette étape est réalisée par un chargement statique de torsion et contrôlée par la corrélation d'images. Ensuite, la seconde étape consiste à réaliser un simple essai de compression sur tube. La mesure du champ de déformations par corrélation permet de visualiser les effets locaux qui perturbent la mesure et conduisent à une rupture précipitée de l'éprouvette. Le protocole d'essai a déjà été validé et présenté dans [16].

\subsection{Résultats}

Les résultats de ces expériences sont synthétisés sur la figure 5. II apparaît d'une part que le comportement en compression sur une éprouvette saine est significativement non-linéaire (Fig. 5a). Cette nonlinéarité a déjà été observée par de nombreux auteurs $[24,34,35,36]$ et provient essentiellement du comportement non-linéaire des fibres de carbone. Une modélisation efficace est proposée par [24] et concorde bien avec nos mesures. Lorsque l'endommagement est introduit dans les éprouvettes, il apparaît que le comportement en terme de rigidité n'est pas affecté. Cela s'explique simplement par le fait que la rigidité est portée quasi-exclusivement par les fibres. Si celles-ci ne sont pas rompues, la rigidité n'est pas modifiée.

En revanche, la baisse de résistance en fonction de l'endommagement est clairement mis en évidence (Figs. 5a et 5b). Plus l'endommagement est important, plus la résistance est faible. Cela concorde bien avec la prédiction des équations (1) ou (2) associées à l'équation (3) c'est-à-dire une rupture en compression proportionnelle à l'endommagement. Un simple modèle linéaire est proposé en déformation pour décrire la baisse de résistance en fonction de l'endom- magement :

Rupture en compression si $\varepsilon<\varepsilon_{\text {min }}^{d}$

$$
\text { où } \quad \varepsilon_{\min }^{d}=\varepsilon_{\min }^{d=0}(1-d)
$$

où $\varepsilon_{\min }^{d=0}$ est la déformation maximale en compression pour un matériau non endommagé. La figure $5 b$ montre la très bonne corrélation entre le modèle et les points expérimentaux (Fig. 5b).

\subsection{Conséquence sur une éprouvette avec défaut d'alignement des fibres}

Il vient d'être montré que l'endommagement affectait significativement la baisse de résistance en compression. II apparaît également que, lors d'un essai avec des fibres présentant un défaut d'alignement, le cisaillement dans la matrice peut devenir critique. La figure 6 présente l'évolution de la variable d'endommagement durant un essai de compression pour différentes valeurs de désalignement. Ces simulations sont effectuées par un modèle éléments finis permettant d'accéder à la valeur de l'endommagement proposé dans $[37,38]$ et implémenté dans ABAQUS.

On observe que plus le désalignement des fibres est important, plus le cisaillement (et donc l'endommagement) est important. Ainsi en prenant en compte le critère de rupture énoncé précédemment (Eq. (4)), il apparaît bien que la rupture sens fibre intervient pour des déformations de plus en plus faibles.

Lorsque le mauvais alignement des fibres devient trop important, c'est directement le cisaillement qui est la cause de la rupture. Une simple écriture des équations d'équilibre sur un pli donne directement l'angle à partir duquel le cisaillement devient critique. Dans le paragraphe suivant, on considèrera un stratifié $[0,90]_{13 s}$ incliné d'un angle $\theta$ (avec $\theta$ petit). Les contraintes dans le repère des plis orientés à $0^{\circ}+\theta$ s'écrivent alors :

$$
\sigma_{11} \approx 2 \sigma_{x x} \quad \sigma_{22} \approx 0 \quad \sigma_{12} \approx \theta \sigma_{x x}
$$

où $\sigma_{x x}$ est la contrainte moyenne appliquée sur le stratifié selon l'axe de l'éprouvette. A partir des données matériaux $\left(\sigma_{11}^{\max }=1461 \mathrm{MPa}, \sigma_{12}^{\max }=112\right.$ $\mathrm{MPa}$ ), on en déduit l'angle pour lequel le cisaillement devient dimensionnant :

$$
\theta_{\max }=2 \frac{180}{\pi} \frac{\sigma_{12}^{\max }}{\sigma_{11}^{\max }}=8,8^{\circ}
$$




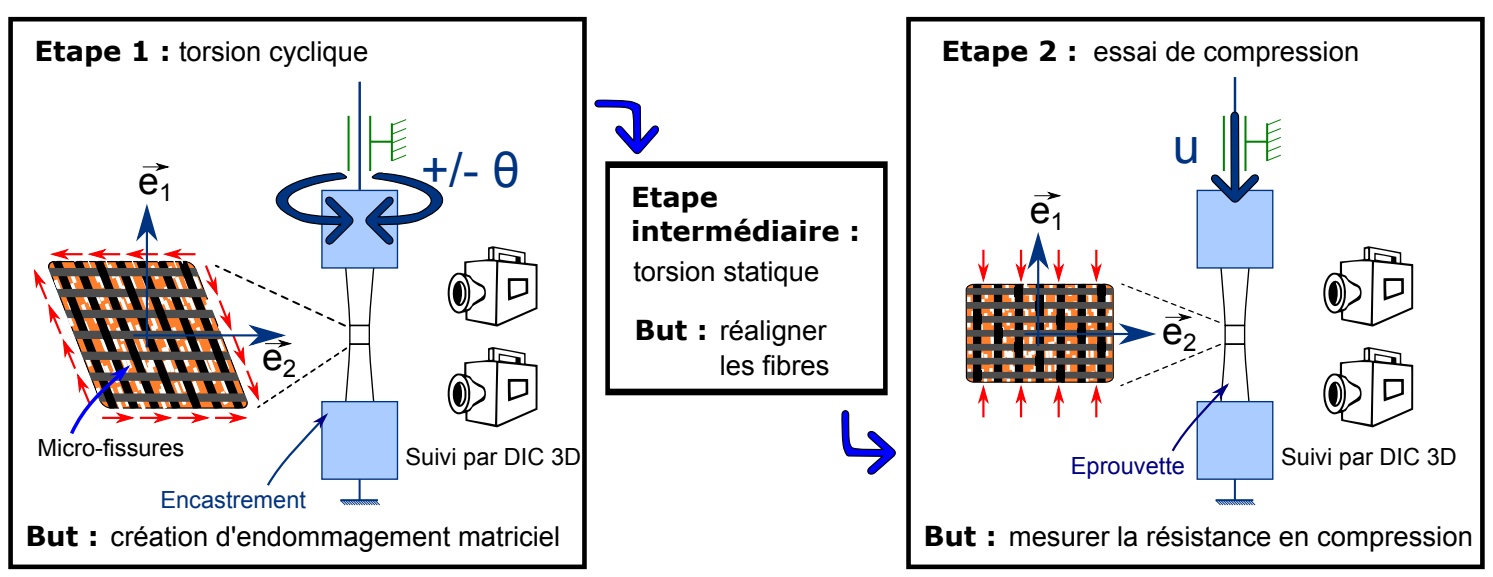

Figure 3 - Protocole de mesure pour quantifier l'effet de l'endommagement sur la rupture en compression sens fibre

Fig. 3. Measuring protocol to quantify the effect of damage on the compressive strength in the fiber direction

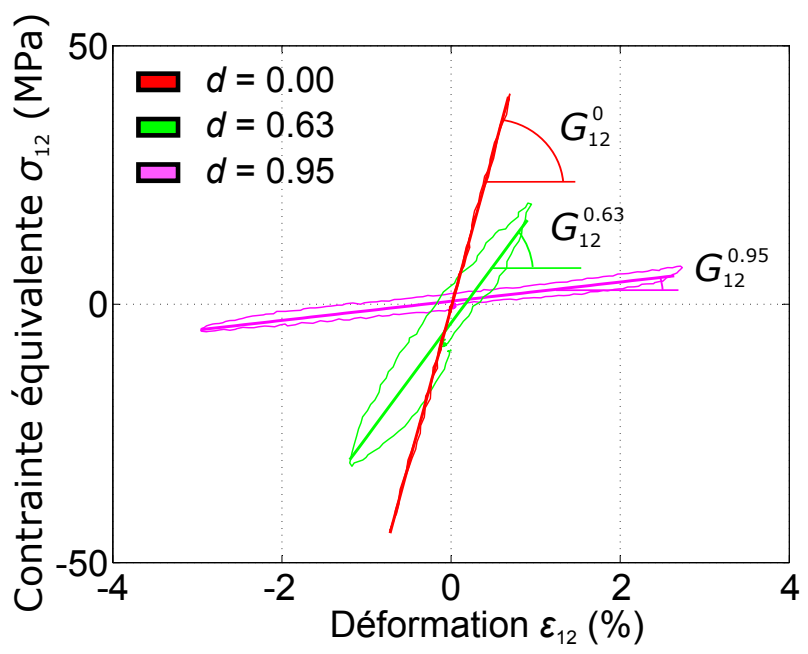

Figure 4 - Mise en évidence de la perte de rigidité en cisaillement : cycle contrainte/déformation du matériau sain $(d=0)$ et après deux niveaux de fatigue en cisaillement $(d=0.63$ et 0.95$)$

Fig. 4. Illustration of the shear stiffness decrease : stress/strain cycle of the undamaged material $(d=0)$ and after two levels of shear fatigue $(d=0.63$ and 0.95$)$

\section{Influence de l'alignement sur la résistance sens fibre}

\subsection{Méthode}

Comme il a été dit dans le paragraphe 2 , les essais de compression pure sont complexes à réaliser. Une solution pour faciliter les expérimentations est de travailler sur des éprouvettes possédant des concentrations de contrainte [39, 40,41]. Dans ce cas, les champs ne sont évidemment plus homogènes et il est connu dans la littérature $[42,37]$ que les contraintes, au niveau de la concentration, peuvent être bien supérieures à la contrainte maximale sens fibre. II est alors nécessaire d'introduire des outils de mécanique de la rupture. Ici on utilisera les critères point stress et average stress [42]. Le premier critère stipule que ce n'est pas la contrainte au bord de la concentration qui pilote la rupture mais plutôt une contrainte à une certaine distance de la concentration, appelée longueur caractéristique. Le second propose plutôt de considérer la moyenne des contraintes le long d'une segment, appelée aussi longueur caractéristique, démarrant au bord de la concentration.

L'essai proposé ici est un essai de compression sur des éprouvettes constituées de plis unidirectionnels positionnés à $0^{\circ}$ et $90^{\circ}$, présentant un élancement faible (faible longueur et épaisseur importante) et une concentration contrainte.

Les éprouvettes sont découpées à partir d'une plaque de 52 plis d'unidirectionnel pré-imprégnés d'épaisseur totale $7.0 \mathrm{~mm}$. L'épaisseur de cette plaque est déterminée afin d'obtenir un grand coefficient de sécurité sur la charge critique de flambage. La résine utilisée est la $M 21$ et la fibre est désignée T700GC. Trois types de concentrations de contrainte sont utilisées : un trou; des encoches à petit rayon 


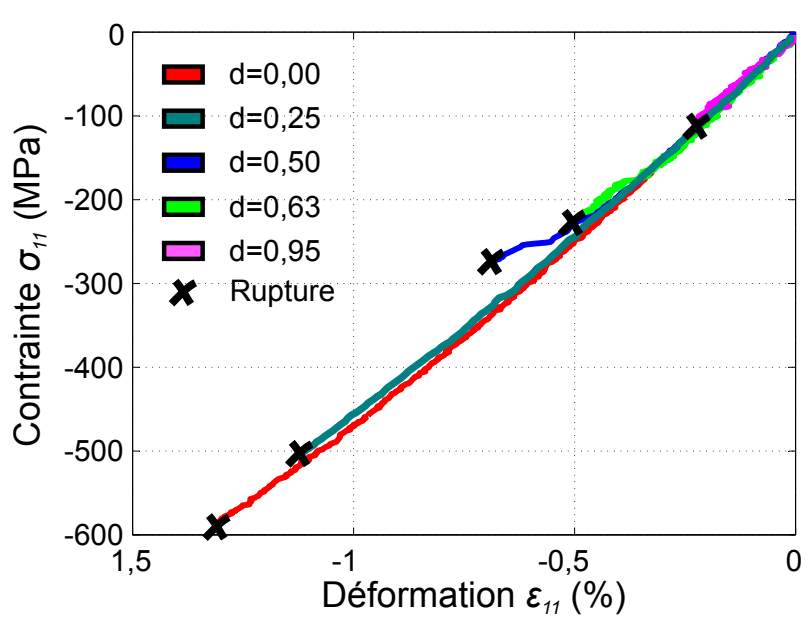

(a) Comportement en compression

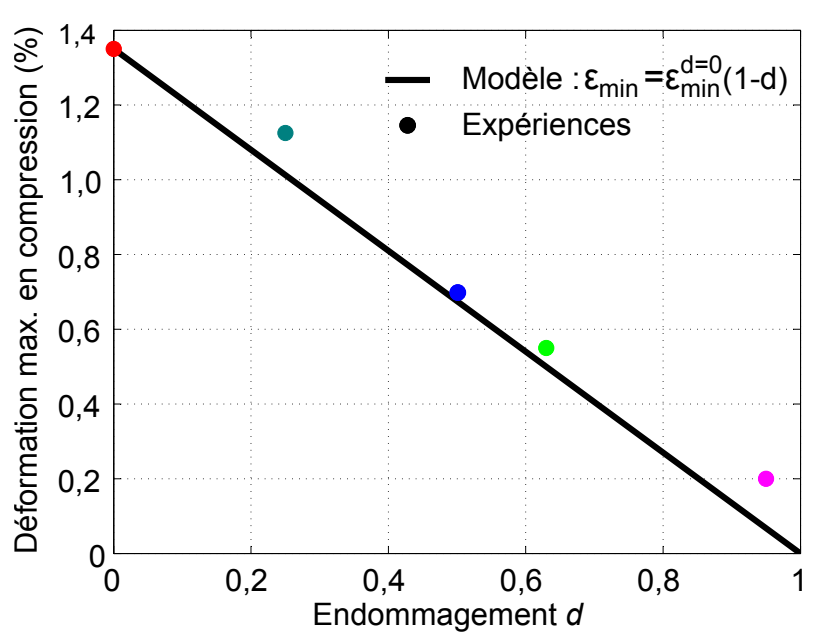

(b) Déformation à rupture en compression

Figure 5 - Effet de l'endommagement sur le comportement en compression sens fibre

Fig. 5. Effect of damage on the compressive behavior in the fiber direction : (a) Compressive behavior; (b) Maximum compressive strain

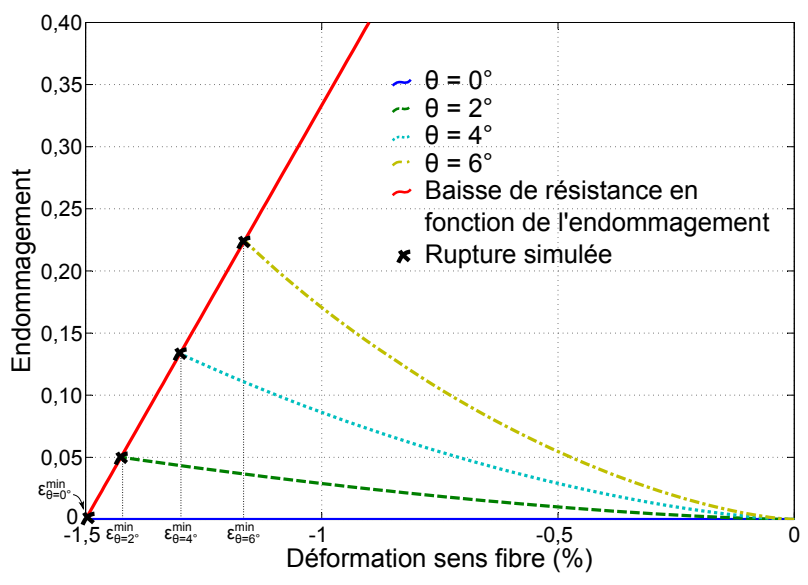

FiguRE 6 - Influence de l'alignement des fibres sur la rupture en compression sens fibre (résultats numériques avec prise en compte de la baisse de résistance en fonction de l'endommagement)

Fig. 6. Influence of fiber misalignment on the compressive strength in the fiber direction (numerical simulations including the drop of compressive strength due to damage)

de courbure et à grand rayon de courbure (forme dite haltère). Leur géométrie est définie sur la figure 7 . Là aussi, les essais sont suivis par une méthode de corrélation d'images.

Notons qu'ici les critères point stress et average stress seront utilisés en déformation car la corrélation d'image donne directement le champ de déformation et que le comportement du matériau en compression est non linéaire (Fig. 5b).

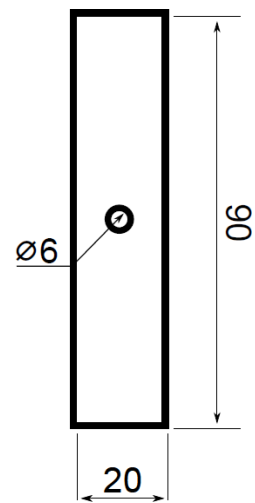

(a)

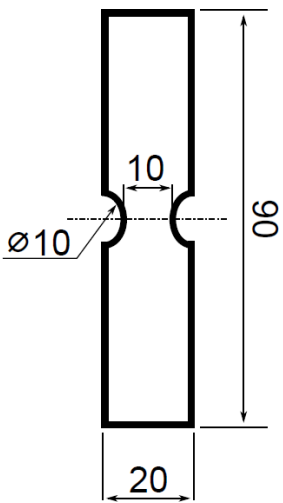

(b)

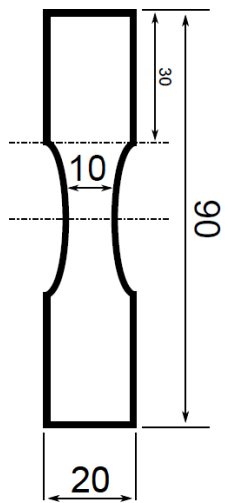

(c)
Figure 7 - Géométrie des éprouvettes avec concentrations de contrainte : (a) éprouvette trouée; (b) éprouvette avec encoches; (c) éprouvette haltère

Fig. 7. Specimens with stress concentrations geometry : (a) specimen with a hole; (b) notched specimen; (c) dumbbell-shaped specimen

\subsection{Résultats sur barreaux sans défaut d'alignement}

Pour pouvoir étudier les barreaux avec défaut d'alignement, il est tout d'abord nécessaire de qualifier les deux critères dans le cas sans défaut. Les essais sont menés jusqu'à la rupture brutale des éprouvettes. La figure 8 montre l'état de trois éprouvettes à la fin de l'essai. La figure 9 correspond à l'évolution de la déformation à proximité de la concentration sur des barreaux avec les différentes concentrations de contraintes. La mesure par corrélation d'images ne permettant pas d'obtenir finement les déformations au bords de la concentration les résultats présentés 


\begin{tabular}{lccc}
\hline Critère & $\begin{array}{c}\text { Point } \\
\text { stress }\end{array}$ & $\begin{array}{c}\text { Average } \\
\text { stress }\end{array}$ \\
\hline Déformation à rupture & $\%$ & $-1,44$ & $-1,44$ \\
\hline Longueur caract. & $\mathrm{mm}$ & 0,4 & 1 \\
\hline
\end{tabular}

TABLE i - Identification des critères de rupture (point stress et average stress) sur barreaux sans défaut d'alignement

Tab. i. Identification of failure criteria (point stress and average stress) on specimens without misalignment defect

sur la figure sont des résultats interpolés. La figure 9a montre bien qu'un dimensionnement à partir de la déformation maximale n'est pas efficace puisque les déformations aux bords du trou sont très différentes suivant la géométrie de l'éprouvette choisie. II apparaît cependant que les trois courbes s'intersectent en un même point ce qui est bien révélateur d'une longueur caractéristique propre au matériau typique d'une méthode point stress.

Ce type de méthode permet donc directement d'accéder à la déformation ultime du matériau en compression sens fibre. La longueur caractéristique s'identifie aisément à partir des trois essais présentés ici. II apparaît que la longueur caractéristique identifiée ici est proche de celle usuellement utilisée en traction [37, 42]. Il est également intéressant de noter que la méthode average stress fonctionne aussi ici. Elle est de plus plus robuste puisque la pente des courbes est moins importante par effet de moyenne.

L'identification des critères de rupture mène donc aux résultats du tableau i. On notera que les deux critères donnent la même déformation à rupture.

\subsection{Résultats sur barreaux avec défaut d'alignement}

L'effet d'un défaut d'alignement est testé sur deux éprouvettes avec de grands rayons de courbure. La première possède une inclinaison de 2,7 degrés et la seconde une inclinaison de 4,7 degrés. La mesure du désalignement est simplement réalisée à partir de la photo de l'éprouvette. Elle correspond à l'angle entre la verticale de l'éprouvette et la direction des fibres. Compte-tenu des résultats précédents, les deux éprouvettes devraient se rompre en compression sens fibre. L'essai est suivi par corrélation d'images. Là aussi, la rupture des éprouvettes est brutale. La valeur de déformation à rupture est obtenue en utilisant une méthode average stress au voisinage de la concentration de contraintes juste avant la rupture. La longueur caractéristique utilisée pour le critère est celle identifiée dans la partie précé- dente. Les résultats de déformations à rupture sont présentés sur la figure 10. II apparaît que la déformation à rupture baisse significativement en fonction de l'orientation des fibres. Le modèle proposé avec baisse de résistance en fonction de l'endommagement est aussi simulé sur la figure. II reste proche des valeurs expérimentales.

Ces résultats ne sont pas directement comparables aux modèles de la micromécanique, en particulier, parce que les éprouvettes étudiées sont constituées de plis à $0^{\circ}$ et $90^{\circ}$, parce que les mesures sont réalisées en déformation et aussi parce que le défaut d'alignement mis en jeu ici est global (échelle méso) et non local (échelle micro) cependant nos expériences montrent un comportement méso très similaire au comportement observé expérimentalement par Piggott et Mrse $[43,44]$ et numériquement par Wisnom [45]. Dans ces études, les auteurs considèrent l'effet des ondulations des mèches de fibres à l'échelle méso. Piggott et Mrse $[43,44]$ observent expérimentalement une décroissance linéaire de la résistance en compression en fonction de l'ondulation. Wisnom [45] montre également via une simulation par éléments finis que cette décroissance n'est pas aussi brutale que les prédictions effectuées par les modèles à l'échelle de la fibre (voir Fig. 2). Son modèle tient notamment compte de la nonlinéarité de comportement du module de cisaillement.

\section{Conclusion}

Ce papier s'est architecturé en plusieurs étapes afin d'étudier l'influence de l'alignement des fibres sur la résistance en compression sens fibre. Dans un premier temps, des résultats issus de $[15,16]$ ont été présentés. Ces résultats montrent que l'endommagement matriciel joue un rôle important dans la résistance des composites en compression sens fibre. Plus précisément, la déformation à rupture décroît linéairement avec la variable d'endommagement $d$.

Dans un second temps, il a été montré, que lors d'un essai de compression avec des fibres présentant un défaut d'alignement, le cisaillement de la matrice pouvait devenir important et générer un endommagement matriciel important. Compte-tenu du modèle préalablement établi, il s'avère donc que ce modèle méso est capable de prendre en compte une baisse de résistance en fonction de l'alignement des fibres.

Une confrontation à l'expérience a ensuite été présentée. II semble que le modèle proposé est relativement concordant avec l'expérience bien qu'il ne modélise pas directement l'influence de l'alignement local des fibres. Ces résultats présentés ici ne prouvent 


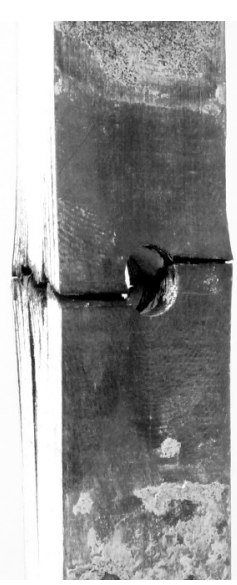

(a)

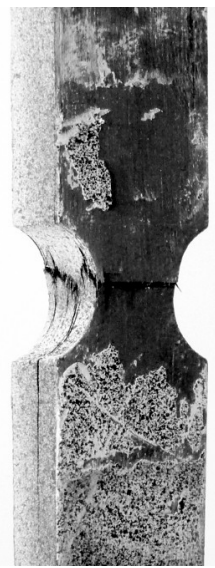

(b)

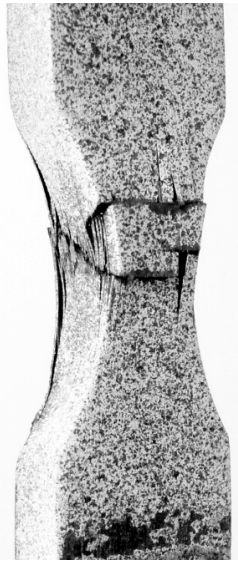

(c)

Figure 8 - État des éprouvettes sans défaut d'alignement après rupture en compression : (a) éprouvette trouée; (b) éprouvette avec encoches; (c) éprouvette haltère

Fig. 8. Specimens without misalignment defect after brutal compressive fracture : (a) specimen with a hole; (b) notched specimen; (c) dumbbell-shaped specimen

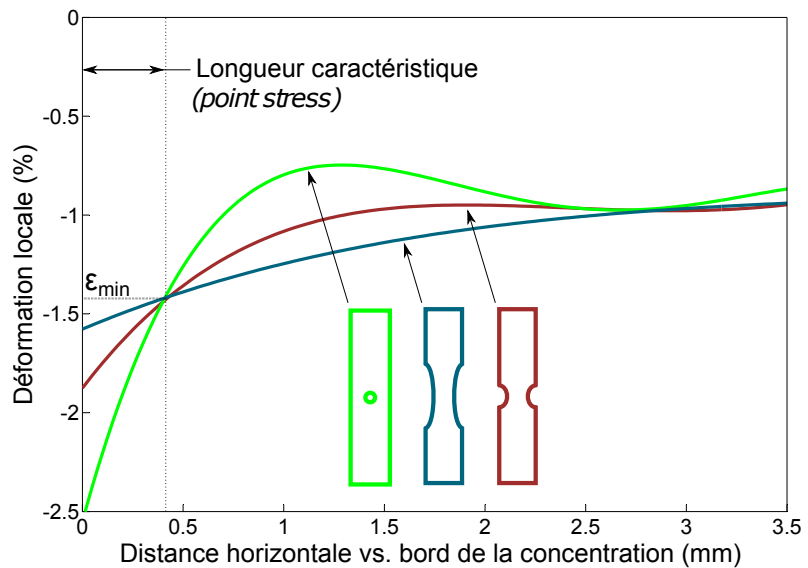

(a) Déformation locale proche du bord (et identification du critère point stress)

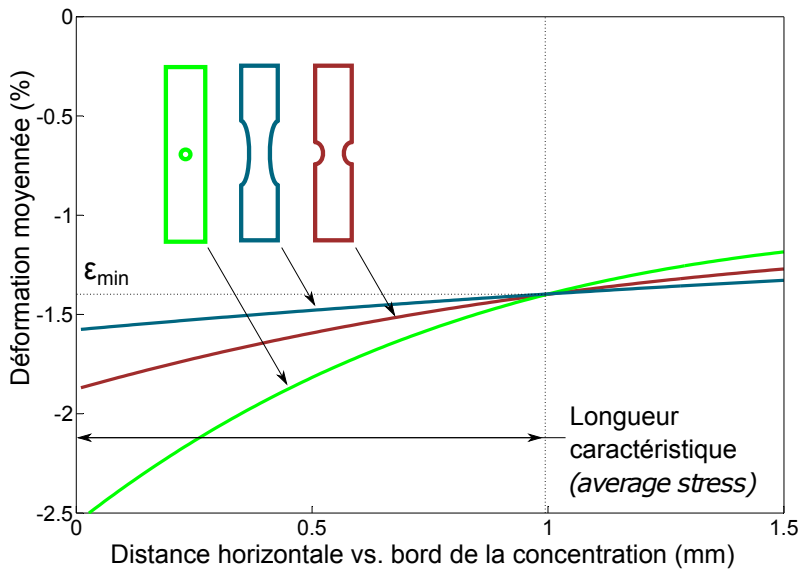

(b) Identification de critère average stress

FiguRE 9 - Méthode de prédiction de la rupture pour une éprouvette avec concentrations de contraintes

Fig. 9. Strength prediction method for specimens with stress concentrations : (a) local strain near the edge and identification with the point stress criterion; (b) identification with the average stress criterion

aucunement que la baisse de résistance observée lors d'un essai de compression avec défaut d'alignement s'explique uniquement par la modélisation proposée. Cependant ces résultats montrent bien qu'il est nécessaire d'intégrer les processus d'endommagement matriciel lors d'un essai de compression avec des fibres désorientées.

\section{Références}

[1] Hochard C., Miot S., Thollon Y., Lahellec N., and Charles J.-P. Fatigue laminated composite structures with stress concentrations. Composites : Part $B$, $65: 11-16,2014$.

[2] A.G. Gibson, M.E. Otheguy Torres, T.N.A. Browne, S. Feih, and A.P. Mouritz. High temperature and fire behaviour of continuous glass fibre/polypropylene laminates. Composites Part A : Applied Science and Manufacturing, 41(9) :1219-1231, September 2010.

[3] B. W. Rosen. Mechanics of composite strenghtening. In Fibre Composite Materials, American Society for metals, pages 37-75. 1964.

[4] B. Budiansky. Micromechanics. Computers \& Structures, 16(1) :3-12, 1983.

[5] B. Budiansky and N. A. Fleck. Compressive failure of fibre composites. Journal of the Mechanics and Physics of Solids, 41(1) :183-211, 1993.

[6] S. Kyriakides, R. Arseculeratne, J. Perry, and K. M. Liechti. On the compressive failure of fiber reinforced composites. International journal of solids and structures, 32(6-7) :689-738, 1995. 


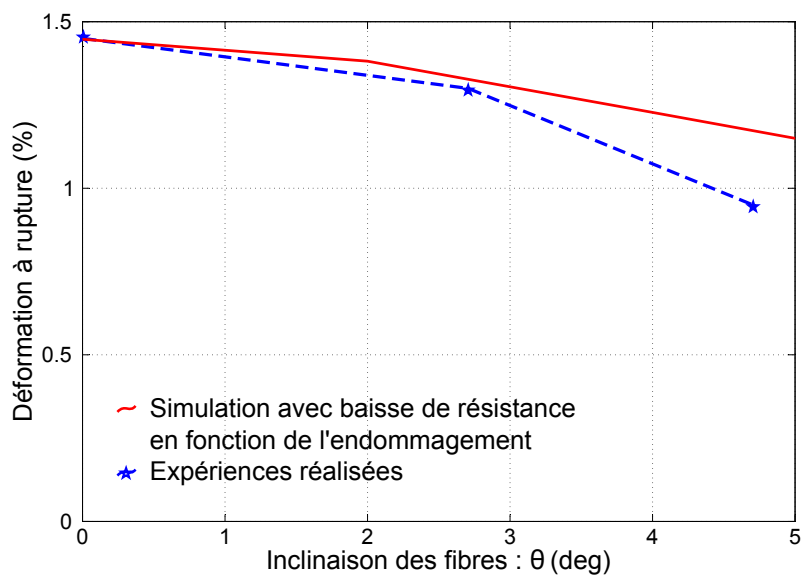

Figure 10 - Influence de l'alignement des fibres sur la rupture en compression sens fibre (résultats expérimentaux)

Fig. 10. Influence of fiber misalignment on the compressive strength in the fiber direction (experimental results)

[7] T. J. Vogler and S. Kyriakides. On the initiation and growth of kink bands in fiber composites : Part i. experiments. International journal of solids and structures, 38(15) :2639-2651, 2001.

[8] N. Feld, O. Allix, E. Baranger, and J. M. Guimard. A micromechanics-based mesomodel for unidirectional laminates in compression. In Proceedings of the 3rd ECCOMAS Thematic Conference on the Mechanical Response of Composites, pages 61-68, 2011.

[9] K. H. Lo and E. S.-M. Chim. Compressive strength of unidirectional composites. Journal of Reinforced Plastics and Composites, 11(8) :838-896, 1992.

[10] E. J. Barbero and J. Tomblin. A damage mechanics model for compression strength of composites. International journal of solids and structures, 33(29) :4379-4393, 1996.

[11] S. Pansart, M. Sinapius, and U. Gabbert. A comprehensive explanation of compression strength differences between various CFRP materials : Micromeso model, predictions, parameter studies. Composites Part A : Applied Science and Manufacturing, 40(4) :376-387, April 2009.

[12] N. A. Fleck and J. Y. Shu. Microbuckle initiation in fibre composites : a finite element study. J. Mech. Phys. Solids, 1995.

[13] M. R. Wisnom. Size effects in the testing of fibrecomposite materials. Composites Science and Technology, 59(13) :1937-1957, 1999.

[14] P Ladeveze and E Ledantec. Damage modelling of the elementary ply for laminated composites. Composites Science and Technology, 43(3) :257-267, 1992.

[15] G. Eyer, O. Montagnier, J-P. Charles, and C. Hochard. Effet de l'endommagement transverse sur la résistance en compression sens fibre d'un composite carbone/époxy. In Journées Nationales des composites 18, 2013.
[16] G. Eyer, O. Montagnier, J-P. Charles, and C. Hochard. Effect of transverse damage on compressive strength in fiber direction for cfrp. In ECCM Seville, 2014.

[17] O Montagnier and C Hochard. Compression characterization of high-modulus carbon fibers. Journal of Composite Materials, 39 :35-49, January 2005.

[18] A. Jumahat, C. Soutis, F.R. Jones, and A. Hodzic. Fracture mechanisms and failure analysis of carbon fibre/toughened epoxy composites subjected to compressive loading. Composite Structures, 92(2) :295305, January 2010.

[19] M. M. Shokrieh and M. J. Omidi. Compressive response of glass-fiber reinforced polymeric composites to increasing compressive strain rates. Composite Structures, 89(4) :517-523, 2009.

[20] D Adams. Current compression test methods. HighPerformance Composites, 2005.

[21] J. Lee and C. Soutis. A study on the compressive strength of thick carbon fibre-epoxy laminates. Composites science and technology, 67(10):2015-2026, 2007.

[22] N. A. Fleck. Compressive failure of fiber composites. Advances in applied mechanics, 33, 1997.

[23] J.-C. Grandidier, P. Casari, and C. Jochum. A fibre direction compressive failure criterion for long fibre laminates at ply scale, including stacking sequence and laminate thickness effects. Composite Structures, 94(12) :3799-3806, December 2012.

[24] O Allix, P Ladevèze, and E Vittecoq. Modelling and identification of the mechanical behaviour of composite laminates in compression. Composites Science and Technology, 1994.

[25] N.V. De Carvalho, S.T. Pinho, and P. Robinson. An experimental study of failure initiation and propagation in 2D woven composites under compression. Composites Science and Technology, 71(10) :13161325, July 2011.

[26] P Ladeveze, Y. Remond, and E. Vittecoq. Essais mécaniques sur composites à hautes performances : difficultés et critères de validité. Bulletin S.F.M., 1989.

[27] S.T. Pinho, P. Robinson, and L. lannucci. Developing a four point bend specimen to measure the mode $\mathrm{i}$ intralaminar fracture toughness of unidirectional laminated composites. Composites Science and Technology, 69(7-8) :1303-1309, June 2009.

[28] M. R. Wisnom and J. W. Atkinson. Constrained buckling tests show increasing compressive strain to failure with increasing strain gradient. Composites Part A : Applied Science and Manufacturing, 28(11) :959-964, 1997.

[29] C. Bois, O. Montagnier, and C. Hochard. Caracterisation of compression behaviour of composite materials using a pure bending test. In Journées Nationales des composites 15, pages 1-8, 2007. 
[30] S. Drapier, C. Gardin, J. C. Grandidier, and M. Potier-Ferry. Structure effect and microbuckling. Composites science and technology, 56(7) :861-867, 1996.

[31] S. Miot. Rupture de structures composites stratifiées sous chargements statique et de fatigue. PhD thesis, Aix-Marseille Université, 2009.

[32] G. Eyer, O. Montagnier, J-P. Charles, and C. Hochard. Design of a composite tube to analyze the compressive behavior of cfrp. Composites Part $A$ : Applied Science and Manufacturing, 87 :115-122, 2016.

[33] ARAMIS. http://www.gom.com, 2015.

[34] O Allix, H Girard, P Ladeveze, and E Vittecoq. Composites $2 \mathrm{D}$ à fibres haute résistance : caractérisation du comportement de compression. In Journées $\mathrm{Na}$ tionales des composites 6, pages 515-526. Ed. Pluralis Paris, 1988.

[35] N. Feld. Vers un pont micro-meso de la rupture en compression des composites stratifiés. PhD thesis, ENS CACHAN, Cachan, 2011.

[36] N.V. De Carvalho, S.T. Pinho, and P. Robinson. Analytical modelling of the compressive and tensile response of woven composites. Composite Structures, 94(9) :2724-2735, September 2012.

[37] C. Hochard, N. Lahellec, and C. Bordreuil. A ply scale non-local fibre rupture criterion for cfrp woven ply laminated structures. Composite Structures, 80(3) :321-326, 2007.

[38] Y. Thollon and C. Hochard. A general damage model for woven fabric composite laminates up to first failure. Mechanics of Materials, 41(7) :820-827, 2009.

[39] N. A. Fleck, P. M. Jelf, and P.T. Curtis. Compressive failure of laminated and woven composites. Journal of Composites Technology and Research, 17(3) :212-220, 1995.

[40] M.J. Laffan, S.T. Pinho, P. Robinson, L. Iannucci, and A.J. McMillan. Measurement of the fracture toughness associated with the longitudinal fibre compressive failure mode of laminated composites. Composites Part A : Applied Science and Manufacturing, 43(11) :1930-1938, November 2012.

[41] S.T. Pinho, P. Robinson, and L. lannucci. Fracture toughness of the tensile and compressive fibre failure modes in laminated composites. Composites Science and Technology, 66(13) :2069-2079, October 2006.

[42] J.M. Whitney and R.J. Nuismer. Stress fracture criteria for laminated composites containing stress concentrations. Journal of composite materials, 8(3) :253-265, 1974.

[43] M. R. Piggott. The effect of fibre waviness on the mechanical properties of unidirectional fibre composites: A review. Composites science and technology, 53(2) :201-205, 1995.

[44] A. M. Mrse and M. R. Piggott. Compressive properties of unidirectional carbon fibres laminates : II. the effects of unintentional and intentional fibre misalignments. Composites science and technology, 46(3) :219-227, 1993.

[45] M. Wisnom. Nonlinear analysis of misaligned unidirectional carbon fibre-epoxy compression specimens. Composites Engineering, 3(6) :547-556, 1993. 